\title{
NEW EXTENSIONS OF THE HERMITE-HADAMARD INEQUALITIES INVOLVING RIEMANN-LIOUVILLE FRACTIONAL INTEGRALS
}

\author{
H. BUDAK, H. KARA, M. Z. SARIKAYA, AND M. E. KIRIŞ
}

Received 15 October, 2019

\begin{abstract}
In this study, we establish the above and below bounds for the left and right hand sides of fractional Hermite-Hadamard inequalities by using functions whose second derivatives are bounded. We also give some refinements of fractional Hermite-Hadamard inequalities by using the functions that have the conditions $f^{\prime}(a+b-t)-f^{\prime}(t) \geq 0, t \in\left[a, \frac{a+b}{2}\right]$.
\end{abstract}

2010 Mathematics Subject Classification: 26D07; 26D10; 26D15; 26A33

Keywords: Hermite-Hadamard inequality, integral inequalities, bounded functions

\section{INTRODUCTION}

The Hermite-Hadamard inequality, which is the first fundamental result for convex mappings with a natural geometrical interpretation and many applications, has drawn attention much interest in elementary mathematics. A number of mathematicians have devoted their efforts to generalise, refine, counterpart and extend it for different classes of functions such as using convex mappings.

The inequalities discovered by C. Hermite and J. Hadamard for convex functions are considerable significant in the literature (see, e.g., [18, p.137], [7]). These inequalities state that if $f: I \rightarrow \mathbb{R}$ is a convex function on the interval $I$ of real numbers and $a, b \in I$ with $a<b$, then

$$
f\left(\frac{a+b}{2}\right) \leq \frac{1}{b-a} \int_{a}^{b} f(x) d x \leq \frac{f(a)+f(b)}{2} .
$$

Both inequalities hold in the reversed direction if $f$ is concave.

In the following we will give some necessary definitions and mathematical preliminaries of fractional calculus theory which are used further in this paper.

Definition 1. Let $f \in L_{1}[a, b]$. The Riemann-Liouville integrals $J_{a+}^{\alpha} f$ and $J_{b-}^{\alpha} f$ of order $\alpha>0$ with $a \geq 0$ are defined by

$$
J_{a+}^{\alpha} f(x)=\frac{1}{\Gamma(\alpha)} \int_{a}^{x}(x-t)^{\alpha-1} f(t) d t, x>a
$$


and

$$
J_{b-}^{\alpha} f(x)=\frac{1}{\Gamma(\alpha)} \int_{x}^{b}(t-x)^{\alpha-1} f(t) d t, x<b
$$

respectively. Here, $\Gamma(\alpha)$ is the Gamma function and $J_{a+}^{0} f(x)=J_{b-}^{0} f(x)=f(x)$.

For more information about fraction calculus please refer to $[9,14,16,19]$.

In [23], Sarikaya et al. first give the following interesting integral inequalities of Hermite-Hadamard type involving Riemann-Liouville fractional integrals.

Theorem 1. Let $f:[a, b] \rightarrow \mathbb{R}$ be a positive function with $0 \leq a<b$ and $f \in$ $L_{1}[a, b]$. If $f$ is a convex function on $[a, b]$, then the following inequalities for fractional integrals hold:

$$
f\left(\frac{a+b}{2}\right) \leq \frac{\Gamma(\alpha+1)}{2(b-a)^{\alpha}}\left[J_{a+}^{\alpha} f(b)+J_{b-}^{\alpha} f(a)\right] \leq \frac{f(a)+f(b)}{2}
$$

with $\alpha>0$.

Sarikaya and Yildirim also give the following Hermite-Hadamard type inequality for the Riemann-Lioville fractional integrals:

Theorem 2 ([24]). Let $f:[a, b] \rightarrow \mathbb{R}$ be a positive function with $a<b$ and $f \in$ $L_{1}[a, b]$. If $f$ is a convex function on $[a, b]$, then the following inequalities for fractional integrals hold:

$$
f\left(\frac{a+b}{2}\right) \leq \frac{2^{\alpha-1} \Gamma(\alpha+1)}{(b-a)^{\alpha}}\left[J_{\left(\frac{a+b}{2}\right)^{+}}^{\alpha} f(b)+J_{\left(\frac{a+b}{2}\right)^{-}}^{\alpha} f(a)\right] \leq \frac{f(a)+f(b)}{2} .
$$

Moreover, Dragomir give the following another version of Hermite-Hadamard inequality for Riemann-Lioville fractional integrals:

Theorem 3 ([8]). Let $f:[a, b] \rightarrow \mathbb{R}$ be a positive function with $a<b$ and $f \in$ $L_{1}[a, b]$. If $f$ is a convex function on $[a, b]$, then the following inequalities for fractional integrals hold:

$$
f\left(\frac{a+b}{2}\right) \leq \frac{2^{\alpha-1} \Gamma(\alpha+1)}{(b-a)^{\alpha}}\left[J_{a^{+}}^{\alpha} f\left(\frac{a+b}{2}\right)+J_{b^{-}}^{\alpha} f\left(\frac{a+b}{2}\right)\right] \leq \frac{f(a)+f(b)}{2}
$$

Over the years several papers devoted to fractional Hermite-Hadamard inequalities. One can refer to the references $[1-4,6,10-13,15,17,20-26]$ for some of them.

F. Chen prove the following inequalities which give the above and below bounds for the left and right hand sides of inequality (1.2).

Theorem 4 ([5]). Let $f:[a, b] \rightarrow \mathbb{R}$ be positive, twice differentiable functions with $a<b$ and $f \in L_{1}[a, b]$. If $f^{\prime \prime}$ is bounded, then we have the inequalities

$$
\frac{m \alpha}{(b-a)^{\alpha}} \int_{a}^{\frac{a+b}{2}}\left(\frac{a+b}{2}-x\right)^{2}\left[(x-a)^{\alpha-1}+(b-x)^{\alpha-1}\right] d x
$$




$$
\begin{aligned}
& \leq \frac{\Gamma(\alpha+1)}{2(b-a)^{\alpha}}\left[J_{a^{+}}^{\alpha} f(b)+J_{b^{-}}^{\alpha} f(a)\right]-f\left(\frac{a+b}{2}\right) \\
& \leq \frac{M \alpha}{(b-a)^{\alpha}} \int_{a}^{\frac{a+b}{2}}\left(\frac{a+b}{2}-x\right)^{2}\left[(x-a)^{\alpha-1}+(b-x)^{\alpha-1}\right] d x
\end{aligned}
$$

and

$$
\begin{aligned}
& \frac{-M \alpha}{2(b-a)^{\alpha}} \int_{a}^{\frac{a+b}{2}}(x-a)(b-x)\left[(x-a)^{\alpha-1}+(b-x)^{\alpha-1}\right] d x \\
& \leq \frac{\Gamma(\alpha+1)}{2(b-a)^{\alpha}}\left[J_{a^{+}}^{\alpha} f(b)+J_{b^{-}}^{\alpha} f(a)\right]-\frac{f(a)+f(b)}{2} \\
& \leq \frac{-m \alpha}{2(b-a)^{\alpha}} \int_{a}^{\frac{a+b}{2}}(x-a)(b-x)\left[(x-a)^{\alpha-1}+(b-x)^{\alpha-1}\right] d x
\end{aligned}
$$

for $\alpha>0$, where $m=\inf _{t \in[a, b]} f^{\prime \prime}(t), M=\sup _{t \in[a, b]} f^{\prime \prime}(t)$.

In this paper we establish extensions of inequalities (1.3) and (1.4).

\section{MAin Results}

Firstly, we give the following inequalities which give the above and below bounds for the left and right hand sides of inequality (1.3).

Theorem 5. Let $f:[a, b] \rightarrow \mathbb{R}$ be positive, twice differentiable functions with $a<b$ and $f \in L_{1}[a, b]$. If $f^{\prime \prime}$ is bounded, i.e. $m \leq f^{\prime \prime}(t) \leq M, t \in[a, b], m, M \in \mathbb{R}$, then we have the inequalities

$$
\begin{aligned}
\frac{m(b-a)^{2}}{4(\alpha+1)(\alpha+2)} & \leq \frac{2^{\alpha-1} \Gamma(\alpha+1)}{(b-a)^{\alpha}}\left[J_{\left(\frac{a+b}{2}\right)^{+}}^{\alpha} f(b)+J_{\left(\frac{a+b}{2}\right)^{-}}^{\alpha} f(a)\right]-f\left(\frac{a+b}{2}\right) \\
& \leq \frac{M(b-a)^{2}}{4(\alpha+1)(\alpha+2)}
\end{aligned}
$$

and

$$
\begin{aligned}
\frac{m(b-a)^{2} \alpha(\alpha+3)}{8(\alpha+1)(\alpha+2)} & \leq \frac{f(a)+f(b)}{2}-\frac{2^{\alpha-1} \Gamma(\alpha+1)}{(b-a)^{\alpha}}\left[J_{\left(\frac{a+b}{2}\right)^{+}}^{\alpha} f(b)+J_{\left(\frac{a+b}{2}\right)^{-}}^{\alpha} f(a)\right] \\
& \leq \frac{M(b-a)^{2} \alpha(\alpha+3)}{8(\alpha+1)(\alpha+2)} .
\end{aligned}
$$


Proof. By using the change of variables we have

$$
\begin{aligned}
& \frac{2^{\alpha-1} \Gamma(\alpha+1)}{(b-a)^{\alpha}}\left[J_{\left(\frac{a+b}{2}\right)^{+}}^{\alpha} f(b)+J_{\left(\frac{a+b}{2}\right)^{-}}^{\alpha} f(a)\right] \\
& =\frac{2^{\alpha-1} \alpha}{(b-a)^{\alpha}}\left[\int_{\frac{a+b}{2}}^{b}(b-x)^{\alpha-1} f(x) d x+\int_{a}^{\frac{a+b}{2}}(x-a)^{\alpha-1} f(x) d x\right] \\
& =\frac{2^{\alpha-1} \alpha}{(b-a)^{\alpha}}\left[\int_{a}^{\frac{a+b}{2}}(b-x)^{\alpha-1} f(a+b-x) d x+\int_{a}^{\frac{a+b}{2}}(x-a)^{\alpha-1} f(x) d x\right] \\
& =\frac{2^{\alpha-1} \alpha}{(b-a)^{\alpha}} \int_{a}^{\frac{a+b}{2}}[f(x)+f(a+b-x)](x-a)^{\alpha-1} d x .
\end{aligned}
$$

By equality (2.3), we get

$$
\begin{aligned}
& \frac{2^{\alpha-1} \Gamma(\alpha+1)}{(b-a)^{\alpha}}\left[J_{\left(\frac{a+b}{2}\right)^{+}}^{\alpha} f(b)+J_{\left(\frac{a+b}{2}\right)^{-}}^{\alpha} f(a)\right]-f\left(\frac{a+b}{2}\right) \\
& =\frac{2^{\alpha-1} \alpha}{(b-a)^{\alpha}} \int_{a}^{\frac{a+b}{2}}[f(x)+f(a+b-x)](x-a)^{\alpha-1} d x-f\left(\frac{a+b}{2}\right) \\
& =\frac{2^{\alpha-1} \alpha}{(b-a)^{\alpha}} \int_{a}^{\frac{a+b}{2}}\left[f(x)+f(a+b-x)-2 f\left(\frac{a+b}{2}\right)\right](x-a)^{\alpha-1} d x .
\end{aligned}
$$

Using the facts that

$$
\begin{gathered}
f(x)-f\left(\frac{a+b}{2}\right)=\int_{\frac{a+b}{2}}^{x} f^{\prime}(t) d t \\
f\left(\frac{a+b}{2}\right)-f(a+b-x)=-\int_{\frac{a+b}{2}}^{a+b-x} f^{\prime}(t) d t
\end{gathered}
$$

we have

$$
f(x)+f(a+b-x)-2 f\left(\frac{a+b}{2}\right)=\int_{\frac{a+b}{2}}^{a+b-x} f^{\prime}(t) d t-\int_{x}^{\frac{a+b}{2}} f^{\prime}(t) d t
$$




$$
\begin{aligned}
& =\int_{x}^{\frac{a+b}{2}} f^{\prime}(a+b-u) d u-\int_{x}^{\frac{a+b}{2}} f^{\prime}(t) d t \\
& =\int_{x}^{\frac{a+b}{2}}\left[f^{\prime}(a+b-t)-f^{\prime}(t)\right] d t .
\end{aligned}
$$

We also have

$$
f^{\prime}(a+b-t)-f^{\prime}(t)=\int_{t}^{a+b-t} f^{\prime \prime}(u) d u .
$$

By using equality (2.6) and $m<f^{\prime \prime}(u)<M, u \in[a, b]$, we obtain,

$$
m \int_{t}^{a+b-t} d u \leq \int_{t}^{a+b-t} f^{\prime \prime}(u) d u \leq M \int_{t}^{a+b-t} d u
$$

i.e.

$$
m(a+b-2 t) \leq f^{\prime}(a+b-t)-f^{\prime}(t) \leq M(a+b-2 t) .
$$

Integrating inequality (2.7) with respect to $t$ on $\left[x, \frac{a+b}{2}\right]$, we get

$$
m\left(\frac{a+b}{2}-x\right)^{2} \leq \int_{x}^{\frac{a+b}{2}}\left[f^{\prime}(a+b-t)-f^{\prime}(t)\right] d t \leq M\left(\frac{a+b}{2}-x\right)^{2} .
$$

By equality (2.5),

$$
m\left(\frac{a+b}{2}-x\right)^{2} \leq f(x)+f(a+b-x)-2 f\left(\frac{a+b}{2}\right) \leq M\left(\frac{a+b}{2}-x\right)^{2} .
$$

Multiplying inequality (2.8) by $\frac{2^{\alpha-1} \alpha(x-a)^{\alpha-1}}{(b-a)^{\alpha}}$ and integrating the resultant inequality with respect to $x$ on $\left[a, \frac{a+b}{2}\right]$, we establish

$$
\begin{aligned}
& \frac{m 2^{\alpha-1} \alpha}{(b-a)^{\alpha}} \int_{a}^{\frac{a+b}{2}}\left(\frac{a+b}{2}-x\right)^{2}(x-a)^{\alpha-1} d x \\
& \leq \frac{2^{\alpha-1} \alpha}{(b-a)^{\alpha}} \int_{a}^{\frac{a+b}{2}}\left[f(x)+f(a+b-x)-2 f\left(\frac{a+b}{2}\right)\right](x-a)^{\alpha-1} d x \\
& \leq \frac{M 2^{\alpha-1} \alpha}{(b-a)^{\alpha}} \int_{a}^{\frac{a+b}{2}}\left(\frac{a+b}{2}-x\right)^{2}(x-a)^{\alpha-1} d x .
\end{aligned}
$$


By using equality (2.4) and

$$
\int_{a}^{\frac{a+b}{2}}\left(\frac{a+b}{2}-x\right)^{2}(x-a)^{\alpha-1} d x=\left(\frac{b-a}{2}\right)^{\alpha+2} \frac{2}{\alpha(\alpha+1)(\alpha+2)},
$$

then we obtain

$$
\begin{aligned}
\frac{m(b-a)^{2}}{4(\alpha+1)(\alpha+2)} & \leq \frac{2^{\alpha-1} \Gamma(\alpha+1)}{(b-a)^{\alpha}}\left[J_{\left(\frac{a+b}{2}\right)^{+}}^{\alpha} f(b)+J_{\left(\frac{a+b}{2}\right)^{-}}^{\alpha} f(a)\right]-f\left(\frac{a+b}{2}\right) \\
& \leq \frac{M(b-a)^{2}}{4(\alpha+1)(\alpha+2)}
\end{aligned}
$$

which completes the proof of inequalities (2.1).

On the other hand, by equality (2.3), we have

$$
\begin{aligned}
& \frac{f(a)+f(b)}{2}-\frac{2^{\alpha-1} \Gamma(\alpha+1)}{(b-a)^{\alpha}}\left[J_{\left(\frac{a+b}{2}\right)^{+}}^{\alpha} f(b)+J_{\left(\frac{a+b}{2}\right)^{-}}^{\alpha} f(a)\right] \\
& =\frac{f(a)+f(b)}{2}-\frac{2^{\alpha-1} \alpha}{(b-a)^{\alpha}} \int_{a}^{\frac{a+b}{2}}[f(x)+f(a+b-x)](x-a)^{\alpha-1} d x \\
& =\frac{2^{\alpha-1} \alpha}{(b-a)^{\alpha}} \int_{a}^{\frac{a+b}{2}}[f(a)+f(b)-f(x)-f(a+b-x)](x-a)^{\alpha-1} d x .
\end{aligned}
$$

By using the equalities

$$
f(x)-f(a)=\int_{a}^{x} f^{\prime}(t) d t
$$

and

$$
f(b)-f(a+b-x)=\int_{a+b-x}^{b} f^{\prime}(t) d t
$$

we get

$$
\begin{aligned}
f(a)+f(b)-f(x)-f(a+b-x) & =\int_{a+b-x}^{b} f^{\prime}(t) d t-\int_{a}^{x} f^{\prime}(t) d t \\
& =\int_{a}^{x} f^{\prime}(a+b-u) d u-\int_{a}^{x} f^{\prime}(t) d t \\
& =\int_{a}^{x}\left[f^{\prime}(a+b-t) d t-f^{\prime}(t)\right] d t .
\end{aligned}
$$


By integrating inequality (2.7) with respect to $t$ on $[a, x]$, we get

$$
m \int_{a}^{x}(a+b-2 t) d t \leq \int_{a}^{x}\left[f^{\prime}(a+b-t)-f^{\prime}(t)\right] d t \leq M \int_{a}^{x}(a+b-2 t) d t .
$$

That is,

$$
m(x-a)(b-x) \leq f(a)+f(b)-f(x)-f(a+b-x) \leq M(x-a)(b-x) .
$$

Multiplying inequality (2.11) by $\frac{2^{\alpha-1} \alpha(x-a)^{\alpha-1}}{(b-a)^{\alpha}}$ and integrating the resultant inequality with respect to $x$ on $\left[a, \frac{a+b}{2}\right]$, we establish

$$
\begin{aligned}
& \frac{m 2^{\alpha-1} \alpha}{(b-a)^{\alpha}} \int_{a}^{\frac{a+b}{2}}(b-x)(x-a)^{\alpha-1} d x \\
& \leq \frac{2^{\alpha-1} \alpha}{(b-a)^{\alpha}} \int_{a}^{\frac{a+b}{2}}[f(a)+f(b)-f(x)-f(a+b-x)](x-a)^{\alpha-1} d x \\
& \leq \frac{M 2^{\alpha-1} \alpha}{(b-a)^{\alpha}} \int_{a}^{\frac{a+b}{2}}(b-x)(x-a)^{\alpha-1} d x,
\end{aligned}
$$

i.e.

$$
\begin{aligned}
\frac{m(b-a)^{2} \alpha(\alpha+3)}{8(\alpha+1)(\alpha+2)} & \leq \frac{f(a)+f(b)}{2}-\frac{2^{\alpha-1} \Gamma(\alpha+1)}{(b-a)^{\alpha}}\left[J_{\left(\frac{a+b}{2}\right)^{+}}^{\alpha} f(b)+J_{\left(\frac{a+b}{2}\right)^{-}}^{\alpha} f(a)\right] \\
& \leq \frac{M(b-a)^{2} \alpha(\alpha+3)}{8(\alpha+1)(\alpha+2)} .
\end{aligned}
$$

which gives inequalities (2.2).

This completes the proof of the theorem.

Now we give the following refinement of inequality (1.3).

Theorem 6. Let $f:[a, b] \rightarrow \mathbb{R}$ be positive, twice differentiable functions with $a<b$ and $f \in L_{1}[a, b]$. If $f^{\prime}(a+b-x) \geq f^{\prime}(x)$ for all $x \in\left[a, \frac{a+b}{2}\right]$, then we have the inequalities

$$
f\left(\frac{a+b}{2}\right) \leq \frac{2^{\alpha-1} \Gamma(\alpha+1)}{(b-a)^{\alpha}}\left[J_{\left(\frac{a+b}{2}\right)^{+}}^{\alpha} f(b)+J_{\left(\frac{a+b}{2}\right)^{-}}^{\alpha} f(a)\right] \leq \frac{f(a)+f(b)}{2} .
$$

Proof. Since $f^{\prime}(a+b-x) \geq f^{\prime}(x)$ for all $x \in\left[a, \frac{a+b}{2}\right]$, by equalities (2.4) and (2.5), we have

$$
\frac{2^{\alpha-1} \Gamma(\alpha+1)}{(b-a)^{\alpha}}\left[J_{\left(\frac{a+b}{2}\right)^{+}}^{\alpha} f(b)+J_{\left(\frac{a+b}{2}\right)^{-}}^{\alpha} f(a)\right]-f\left(\frac{a+b}{2}\right)
$$




$$
\begin{aligned}
& =\frac{2^{\alpha-1} \alpha}{(b-a)^{\alpha}} \int_{a}^{\frac{a+b}{2}}\left[f(x)+f(a+b-x)-2 f\left(\frac{a+b}{2}\right)\right](x-a)^{\alpha-1} d x \\
& =\frac{2^{\alpha-1} \alpha}{(b-a)^{\alpha}} \int_{a}^{\frac{a+b}{2}}\left[\int_{x}^{\frac{a+b}{2}}\left[f^{\prime}(a+b-t)-f^{\prime}(t)\right] d t\right](x-a)^{\alpha-1} d x \\
& \geq 0
\end{aligned}
$$

which gives the first inequality in (2.12).

Similarly, by equalities (2.9) and (2.10), we get

$$
\begin{aligned}
& \frac{f(a)+f(b)}{2}-\frac{2^{\alpha-1} \Gamma(\alpha+1)}{(b-a)^{\alpha}}\left[J_{\left(\frac{a+b}{2}\right)^{+}}^{\alpha} f(b)+J_{\left(\frac{a+b}{2}\right)^{-}}^{\alpha} f(a)\right] \\
& =\frac{2^{\alpha-1} \alpha}{(b-a)^{\alpha}} \int_{a}^{\frac{a+b}{2}}[f(a)+f(b)-f(x)-f(a+b-x)](x-a)^{\alpha-1} d x \\
& =\frac{2^{\alpha-1} \alpha}{(b-a)^{\alpha}} \int_{a}^{\frac{a+b}{2}}\left[\int_{a}^{x}\left[f^{\prime}(a+b-t) d t-f^{\prime}(t)\right] d t\right](x-a)^{\alpha-1} d x \\
& \geq 0 .
\end{aligned}
$$

This completes the proof.

Now, we establish the following inequalities which give the above and below bounds for the left and right hand sides of inequality (1.4).

Theorem 7. Let $f:[a, b] \rightarrow \mathbb{R}$ be positive, twice differentiable functions with $a<b$ and $f \in L_{1}[a, b]$. If $f^{\prime \prime}$ is bounded, i.e. $m \leq f^{\prime \prime}(t) \leq M, t \in[a, b], m, M \in \mathbb{R}$, then we have the inequalities

$$
\begin{aligned}
\frac{m(b-a)^{2} \alpha}{8(\alpha+2)} & \leq \frac{2^{\alpha-1} \Gamma(\alpha+1)}{(b-a)^{\alpha}}\left[J_{a^{+}}^{\alpha} f\left(\frac{a+b}{2}\right)+J_{b^{-}}^{\alpha} f\left(\frac{a+b}{2}\right)\right]-f\left(\frac{a+b}{2}\right) \\
& \leq \frac{m(b-a)^{2} \alpha}{8(\alpha+2)}
\end{aligned}
$$

and

$$
\begin{aligned}
\frac{m(b-a)^{2}}{4(a+2)} & \leq \frac{f(a)+f(b)}{2}-\frac{2^{\alpha-1} \Gamma(\alpha+1)}{(b-a)^{\alpha}}\left[J_{a^{+}}^{\alpha} f\left(\frac{a+b}{2}\right)+J_{b^{-}}^{\alpha} f\left(\frac{a+b}{2}\right)\right] \\
& \leq \frac{m(b-a)^{2}}{4(a+2)}
\end{aligned}
$$


Proof. From the definition of Riemann-Liouville fractional integrals, we get

$$
\begin{aligned}
& \frac{2^{\alpha-1} \Gamma(\alpha+1)}{(b-a)^{\alpha}}\left[J_{a^{+}} f\left(\frac{a+b}{2}\right)+J_{b^{-}} f\left(\frac{a+b}{2}\right)\right] \\
& =\frac{2^{\alpha-1} \alpha}{(b-a)^{\alpha}} \int_{a}^{\frac{a+b}{2}}\left(\frac{a+b}{2}-x\right)^{\alpha-1} f(x) d x+\int_{\frac{a+b}{2}}^{b}\left(x-\frac{a+b}{2}\right)^{\alpha-1} f(x) d x \\
& =\frac{2^{\alpha-1} \alpha}{(b-a)^{\alpha}} \int_{a}^{\frac{a+b}{2}}[f(x)+f(a+b-x)]\left(\frac{a+b}{2}-x\right)^{\alpha-1} d x .
\end{aligned}
$$

By equality (2.15), we have

$$
\begin{aligned}
& \frac{2^{\alpha-1} \Gamma(\alpha+1)}{(b-a)^{\alpha}}\left[J_{a^{+}}^{\alpha} f\left(\frac{a+b}{2}\right)+J_{b^{-}}^{\alpha} f\left(\frac{a+b}{2}\right)\right]-f\left(\frac{a+b}{2}\right) \\
& =\frac{2^{\alpha-1} \alpha}{(b-a)^{\alpha}} \int_{a}^{\frac{a+b}{2}}[f(x)+f(a+b-x)]\left(\frac{a+b}{2}-x\right)^{\alpha-1} d x-f\left(\frac{a+b}{2}\right) \\
& =\frac{2^{\alpha-1} \alpha}{(b-a)^{\alpha}} \int_{a}^{\frac{a+b}{2}}\left[f(x)+f(a+b-x)-2 f\left(\frac{a+b}{2}\right)\right]\left(\frac{a+b}{2}-x\right)^{\alpha-1} d x .
\end{aligned}
$$

Moreover, using the identities

$$
f\left(\frac{a+b}{2}\right)-f(x)=\int_{x}^{\frac{a+b}{2}} f^{\prime}(t) d t
$$

and

$$
f(a+b-x)-f\left(\frac{a+b}{2}\right)=\int_{\frac{a+b}{2}}^{a+b-x} f^{\prime}(t) d t
$$

we obtain

$$
\begin{aligned}
f(x)+f(a+b-x)-2 f\left(\frac{a+b}{2}\right) & =\int_{\frac{a+b}{2}}^{a+b-x} f^{\prime}(t) d t-\int_{x}^{\frac{a+b}{2}} f^{\prime}(t) d t \\
& =\int_{x}^{\frac{a+b}{2}} f^{\prime}(a+b-u) d u-\int_{x}^{\frac{a+b}{2}} f^{\prime}(t) d t
\end{aligned}
$$




$$
=\int_{x}^{\frac{a+b}{2}}\left[f^{\prime}(a+b-t)-f^{\prime}(t)\right] d t .
$$

By integrating inequality (2.7) with respect to $t$ on $\left[x, \frac{a+b}{2}\right]$, we get

$$
m\left(\frac{a+b}{2}-x\right)^{2} \leq \int_{x}^{\frac{a+b}{2}}\left[f^{\prime}(a+b-t)-f^{\prime}(t)\right] d t \leq M\left(\frac{a+b}{2}-x\right)^{2} .
$$

That is, by equality (2.17),

$$
m\left(\frac{a+b}{2}-x\right)^{2} \leq f(x)+f(a+b-x)-2 f\left(\frac{a+b}{2}\right) \leq M\left(\frac{a+b}{2}-x\right)^{2} .
$$

Multiplying inequality (2.18) by $\frac{2^{\alpha-1} \alpha(x-a)^{\alpha-1}}{(b-a)^{\alpha}}$ and integrating the resultant inequality with respect to $x$ on $\left[a, \frac{a+b}{2}\right]$, we establish

$$
\begin{aligned}
& \frac{m 2^{\alpha-1} \alpha}{(b-a)^{\alpha}} \int_{a}^{\frac{a+b}{2}}\left(\frac{a+b}{2}-x\right)^{\alpha+1} d x \\
& \leq \frac{2^{\alpha-1} \alpha}{(b-a)^{\alpha}} \int_{a}^{\frac{a+b}{2}}\left[f(x)+f(a+b-x)-2 f\left(\frac{a+b}{2}\right)\right]\left(\frac{a+b}{2}-x\right)^{\alpha-1} d x \\
& \leq \frac{M 2^{\alpha-1} \alpha}{(b-a)^{\alpha}} \int_{a}^{\frac{a+b}{2}}\left(\frac{a+b}{2}-x\right)^{\alpha+1} d x .
\end{aligned}
$$

It follows that

$$
\begin{aligned}
\frac{m(b-a)^{2} \alpha}{8(\alpha+2)} & \leq \frac{2^{\alpha-1} \Gamma(\alpha+1)}{(b-a)^{\alpha}}\left[J_{a^{+}}^{\alpha} f\left(\frac{a+b}{2}\right)+J_{b^{-}}^{\alpha} f\left(\frac{a+b}{2}\right)\right]-f\left(\frac{a+b}{2}\right) \\
& \leq \frac{m(b-a)^{2} \alpha}{8(\alpha+2)}
\end{aligned}
$$

which proves inequality (2.13).

On the other hand, using identity (2.15), we get

$$
\begin{aligned}
& \frac{f(a)+f(b)}{2}-\frac{2^{\alpha-1} \Gamma(\alpha+1)}{(b-a)^{\alpha}}\left[J_{a^{+}}^{\alpha} f\left(\frac{a+b}{2}\right)+J_{b^{-}}^{\alpha} f\left(\frac{a+b}{2}\right)\right] \\
& =\frac{f(a)+f(b)}{2}-\frac{2^{\alpha-1} \alpha}{(b-a)^{\alpha}} \int_{a}^{\frac{a+b}{2}}[f(x)+f(a+b-x)]\left(\frac{a+b}{2}-x\right)^{\alpha-1} d x
\end{aligned}
$$




$$
=\frac{2^{\alpha-1} \alpha}{(b-a)^{\alpha}} \int_{a}^{\frac{a+b}{2}}[f(a)+f(b)-f(x)-f(a+b-x)]\left(\frac{a+b}{2}-x\right)^{\alpha-1} d x .
$$

Using the facts that

$$
f(x)-f(a)=\int_{a}^{x} f^{\prime}(t) d t
$$

and

$$
f(b)-f(a+b-x)=\int_{a+b-x}^{b} f^{\prime}(t) d t
$$

we get

$$
\begin{aligned}
f(a)+f(b)-f(x)-f(a+b-x) & =\int_{a+b-x}^{b} f^{\prime}(t) d t-\int_{a}^{x} f^{\prime}(t) d t \\
& =\int_{a}^{x} f^{\prime}(a+b-u) d u-\int_{a}^{x} f^{\prime}(t) d t \\
& =\int_{a}^{x}\left[f^{\prime}(a+b-t) d t-f^{\prime}(t)\right] d t .
\end{aligned}
$$

By integrating inequality (2.7) with respect to $t$ on $[a, x]$, we get

$$
\begin{aligned}
m\left[\left(\frac{b-a}{2}\right)^{2}-\left(\frac{a+b}{2}-x\right)^{2}\right] & \leq f(a)+f(b)-f(x)-f(a+b-x) \\
& \leq M\left[\left(\frac{b-a}{2}\right)^{2}-\left(\frac{a+b}{2}-x\right)^{2}\right] .
\end{aligned}
$$

Multiplying inequality (2.21) by $\frac{2^{\alpha-1} \alpha}{(b-a)^{\alpha}}\left(\frac{a+b}{2}-x\right)^{\alpha-1}$ and integrating the resultant inequality with respect to $x$ on $\left[a, \frac{a+b}{2}\right]$, we establish

$$
\begin{aligned}
& \frac{m 2^{\alpha-1} \alpha}{(b-a)^{\alpha}} \int_{a}^{\frac{a+b}{2}}\left[\left(\frac{b-a}{2}\right)^{2}-\left(\frac{a+b}{2}-x\right)^{2}\right]\left(\frac{a+b}{2}-x\right)^{\alpha-1} d x \\
& \leq \frac{2^{\alpha-1} \alpha}{(b-a)^{\alpha}} \int_{a}^{\frac{a+b}{2}}[f(a)+f(b)-f(x)-f(a+b-x)]\left(\frac{a+b}{2}-x\right)^{\alpha-1} d x
\end{aligned}
$$




$$
\leq \frac{M 2^{\alpha-1} \alpha}{(b-a)^{\alpha}} \int_{a}^{\frac{a+b}{2}}\left[\left(\frac{b-a}{2}\right)^{2}-\left(\frac{a+b}{2}-x\right)^{2}\right]\left(\frac{a+b}{2}-x\right)^{\alpha-1} .
$$

By equality (2.19) and equality

$$
\frac{2^{\alpha-1} \alpha}{(b-a)^{\alpha}} \int_{a}^{\frac{a+b}{2}}\left[\left(\frac{b-a}{2}\right)^{2}-\left(\frac{a+b}{2}-x\right)^{2}\right]\left(\frac{a+b}{2}-x\right)^{\alpha-1} d x=\frac{(b-a)^{2}}{4(a+2)},
$$

we get the desired inequality (2.14).

This completes the proof.

Theorem 8. Let $f:[a, b] \rightarrow \mathbb{R}$ be positive, twice differentiable functions with $a<b$ and $f \in L_{1}[a, b]$. If $f^{\prime}(a+b-x) \geq f^{\prime}(x)$ for all $x \in\left[a, \frac{a+b}{2}\right]$, then we have the inequalities

$$
f\left(\frac{a+b}{2}\right) \leq \frac{2^{\alpha-1} \Gamma(\alpha+1)}{(b-a)^{\alpha}}\left[J_{a^{+}}^{\alpha} f\left(\frac{a+b}{2}\right)+J_{b^{-}}^{\alpha} f\left(\frac{a+b}{2}\right)\right] \leq \frac{f(a)+f(b)}{2} .
$$

Proof. From equalities (2.16) and (2.17), we have

$$
\begin{aligned}
& \frac{2^{\alpha-1} \Gamma(\alpha+1)}{(b-a)^{\alpha}}\left[J_{a^{+}}^{\alpha} f\left(\frac{a+b}{2}\right)+J_{b^{-}}^{\alpha} f\left(\frac{a+b}{2}\right)\right]-f\left(\frac{a+b}{2}\right) \\
& =\frac{2^{\alpha-1} \alpha}{(b-a)^{\alpha}} \int_{a}^{\frac{a+b}{2}}\left[f(x)+f(a+b-x)-2 f\left(\frac{a+b}{2}\right)\right]\left(\frac{a+b}{2}-x\right)^{\alpha-1} d x \\
& =\frac{2^{\alpha-1} \alpha}{(b-a)^{\alpha}} \int_{a}^{\frac{a+b}{2}}\left[\int_{x}^{\frac{a+b}{2}}\left[f^{\prime}(a+b-t)-f^{\prime}(t)\right] d t\right]\left(\frac{a+b}{2}-x\right)^{\alpha-1} d x \\
& \geq 0
\end{aligned}
$$

which proves the first inequality in (2.22).

Similarly, by equalities (2.19) and (2.20)

$$
\begin{aligned}
& \frac{f(a)+f(b)}{2}-\frac{2^{\alpha-1} \Gamma(\alpha+1)}{(b-a)^{\alpha}}\left[J_{a^{+}}^{\alpha} f\left(\frac{a+b}{2}\right)+J_{b^{-}}^{\alpha} f\left(\frac{a+b}{2}\right)\right] \\
& =\frac{2^{\alpha-1} \alpha}{(b-a)^{\alpha}} \int_{a}^{\frac{a+b}{2}}[f(a)+f(b)-f(x)-f(a+b-x)]\left(\frac{a+b}{2}-x\right)^{\alpha-1} d x \\
& =\frac{2^{\alpha-1} \alpha}{(b-a)^{\alpha}} \int_{a}^{\frac{a+b}{2}}\left[\int_{a}^{x}\left[f^{\prime}(a+b-t) d t-f^{\prime}(t)\right] d t\right]\left(\frac{a+b}{2}-x\right)^{\alpha-1} d x
\end{aligned}
$$




$$
\geq 0 \text {. }
$$

This completes the proof.

\section{REFERENCES}

[1] M. U. Awan, M. A. Noor, T. S. Du, and K. I. Noor, "New refinements of fractional HermiteHadamard inequality," Revista de la Real Academia de Ciencias Exactas, Físicas y Naturales. Serie A. Matemáticas, vol. 113, no. 1, pp. 21-29, 2019, doi: doi.org/10.1007/s13398-017-0448-x.

[2] H. Budak, "On Fejér type inequalities for convex mappings utilizing fractional integrals of a function with respect to another function," Results in Mathematics, vol. 74, no. 1, p. 29, 2019, doi: doi.org/10.1007/s00025-019-0960-8.

[3] H. Budak, "On refinements of Hermite-Hadamard type inequalities for Riemann-Liouville fractional integral operators," An International Journal of Optimization and Control: Theories \& Applications (IJOCTA), vol. 9, no. 1, pp. 41-48, 2019, doi: doi.org/10.11121/ijocta.01.2019.00585.

[4] H. Budak, M. Z. Sarıkaya, and M. K. Yıldız, "Hermite-Hadamard type inequalities for f-convex function involving fractional integrals," Filomat, vol. 32, no. 16, pp. 5509-5518, 2018, doi: doi.org/10.2298/FIL1816509B.

[5] F. Chen, "Extensions of the Hermite-Hadamard inequality for convex functions via fractional integrals," J. Math. Inequal, vol. 10, no. 1, pp. 75-81, 2016, doi: doi:10.7153/jmi-10-07.

[6] F. Chen, "On the generalization of some Hermite-Hadamard inequalities for functions with convex absolute values of the second derivatives via fractional integrals," Ukrainian Mathematical Journal, vol. 12, no. 70, pp. 1953-1965, 2019, doi: doi:10.1007/s11253-019-01618-7.

[7] S. S. Dragomir and C. Pearce, "Selected topics on Hermite-Hadamard inequalities and applications," Mathematics Preprint Archive, vol. 2003, no. 3, pp. 463-817, 2003.

[8] S. S. Dragomir, "Some inequalities of Hermite-Hadamard type for symmetrized convex functions and Riemann-Liouville fractional integrals," RGMIA Res. Rep. Coll, vol. 20, p. 15, 2017.

[9] R. Gorenflo and F. Mainardi, Fractional calculus: integral and differential equations of fractional order, 2008.

[10] A. Gozpinar, E. Set, and S. S. Dragomir, "Some generalized Hermite-Hadamard type inequalities involving fractional integral operator for functions whose second derivatives in absolute value are s-convex," Acta Mathematica Universitatis Comenianae, vol. 88, no. 1, pp. 87-100, 2019.

[11] S.-R. Hwang and K.-L. Tseng, "New Hermite-Hadamard-type inequalities for fractional integrals and their applications," Revista de la Real Academia de Ciencias Exactas, Físicas y Naturales. Serie A. Matemáticas, vol. 112, no. 4, pp. 1211-1223, 2018, doi: doi.org/10.1007/s13398-0170419-2.

[12] M. Jleli and B. Samet, "On Hermite-Hadamard type inequalities via fractional integrals of a function with respect to another function," J. Nonlinear Sci. Appl, vol. 9, no. 3, pp. 1252-1260, 2016.

[13] M. A. Khan, A. Iqbal, M. Suleman, and Y.-M. Chu, "Hermite-Hadamard type inequalities for fractional integrals via Green's function,” Journal of inequalities and applications, vol. 2018, no. 1, p. 161, 2018, doi: doi.org/10.1186/s13660-018-1751-6.

[14] A. A. Kilbas, H. M. Srivastava, and J. J. Trujillo, Theory and applications of fractional differential equations. elsevier, 2006, vol. 204.

[15] K. Liu, J. Wang, and D. O'Regan, "On the Hermite-Hadamard type inequality for $\psi$-RiemannLiouville fractional integrals via convex functions," Journal of Inequalities and Applications, vol. 2019, no. 1, p. 27, 2019, doi: doi.org/10.1186/s13660-019-1982-1.

[16] K. S. Miller and B. Ross, An introduction to the fractional calculus and fractional differential equations. Wiley, 1993. 
[17] P. O. Mohammed and M. Z. Sarikaya, "Hermite-Hadamard type inequalities for f-convex function involving fractional integrals," Journal of inequalities and applications, vol. 2018, no. 1, pp. 1-33, 2018, doi: doi.org/10.1186/s13660-018-1950-1.

[18] J. E. Peajcariaac and Y. L. Tong, Convex functions, partial orderings, and statistical applications. Academic Press, 1992.

[19] I. Podlubny, Fractional differential equations: an introduction to fractional derivatives, fractional differential equations, to methods of their solution and some of their applications. Elsevier, 1998.

[20] S. Qaisar, M. Iqbal, S. Hussain, S. I. Butt, and M. A. Meraj, "New inequalities on HermiteHadamard utilizing fractional integrals," Kragujevac Journal of Mathematics, vol. 42, no. 1, pp. 15-27, 2018.

[21] K. Qiu and J. R. Wang, "A fractional integral identity and its application to fractional HermiteHadamard type inequalities," Journal of Interdisciplinary Mathematics, vol. 21, no. 1, pp. 1-16, 2018, doi: doi.org/10.1080/09720502.2017.1400795.

[22] M. Z. Sarikaya and N. Aktan, "On the generalization some integral inequalities and their applications," Mathematical and Computer Modelling, vol. 54, pp. 9-10.

[23] M. Z. Sarikaya, E. Set, H. Yaldiz, and N. Başak, "Hermite-Hadamard's inequalities for fractional integrals and related fractional inequalities," Mathematical and Computer Modelling, vol. 57, no. 9-10, pp. 2403-2407, 2013, doi: doi.org/10.1016/j.mcm.2011.12.048.

[24] M. Z. Sarikaya and H. Yildirim, "On Hermite-Hadamard type inequalities for Riemann-Liouville fractional integrals," Miskolc Mathematical Notes, vol. 17, no. 2, pp. 1049-1059, 2016, doi: doi.org/10.18514/MMN.2017.1197.

[25] E. Set, A. O. Akdemir, and B. Çelik, "On generalization of Fejér type inequalities via fractional integral operators," Filomat, vol. 32, no. 16, pp. 5537-5547, 2018, doi: doi.org/10.2298/FIL1816537S.

[26] T. Tunç, S. Sönmezoğlu, and M. Z. Sarıkaya, "On integral inequalities of Hermite-Hadamard type via Green function and applications," Applications \& Applied Mathematics, vol. 14, no. 1, 2019.

Authors' addresses

H. Budak

Department of Mathematics, Faculty of Science and Arts, Düzce University, Düzce-Turkey

E-mail address: hsyn.budakegmail.com

H. Kara

Department of Mathematics, Graduate School of Natural and Applied Sciences, Afyon Kocatepe University, Afyon-Turkey

E-mail address: hasan64kara@gmail.com

\section{Z. Sarikaya}

Department of Mathematics, Faculty of Science and Arts, Düzce University, Düzce-Turkey

E-mail address: sarikayamz@gmail.com

\section{E. Kiriș}

Department of Mathematics, Graduate School of Natural and Applied Sciences, Afyon Kocatepe University, Afyon-Turkey

E-mail address: mkiris@gmail.com 